\title{
Extraction of globular clusters members with Gaia DR2 astrometry
}

\author{
Iván H. Bustos Fierro $^{1 \star}$ and J. H. Calderón ${ }^{1,2} \dagger$ \\ ${ }^{1}$ Universidad Nacional de Córdoba. Observatorio Astronómico. Córdoba, Argentina. \\ ${ }^{2}$ Consejo Nacional de Investigaciones Científicas y Técnicas (CONICET), Argentina.
}

Accepted XXX. Received YYY; in original form ZZZ

\begin{abstract}
In this work we present a method to identify possible members of globular clusters using data from Gaia DR2. The method consists of two stages: the first one based on a clustering algorithm, and the second one based on the analysis of the projected spatial distribution of stars with different proper motions. In order to confirm that the clusters members extracted by the method correspond to actual globular clusters, the spatial distribution, the vector point diagram of the proper motions and the colourmagnitude diagrams are analysed. We apply the developed method to eight clusters: NGC 1261, NGC 3201, NGC 6139, NGC 6205, NGC 6362, NGC 6397, NGC 6712 and Palomar 13; we show the number of members extracted, the mean proper motions derived from them and finally we compare our results with other authors. In order to analyse the efficiency of the extraction method we perform an estimation of the completeness and the degree of contamination of the extracted members.
\end{abstract}

Key words: astrometry - globular clusters: general - globular clusters: individual: NGC 1261, NGC 3201, NGC 6139, NGC 6205, NGC 6362, NGC 6397, NGC 6712, Pal 13

\section{INTRODUCTION}

Since stellar clusters are groups of gravitationally bound stars, their members are expected to occupy a rather small volume of space and to share their kinematic properties. Therefore, when space positions and velocities of stars are measured in a region of the sky containing a stellar cluster, field stars (foreground and background) will show a uniform distribution in the sky while the stars in the cluster will lie in a small region. In velocity space the field stars will show a well determined random distribution if the field is rich and a more spread and irregular distribution if the number of them is small, instead, the cluster stars always lie concentrated in a very small region. Based on these elementary characteristics, most galactic clusters have been primarily detected by an overdensity of the projected positions of stars on the sky. Several methods using also proper motions information were developed in order to separate the true members of the cluster from the field stars. Some on them are parametric methods -e.g. Vasilevskis et al. (1965) - and others are nonparametric -e.g. Galadí-Enríquez et al. (1998)- that have been applied on open and globular clusters.

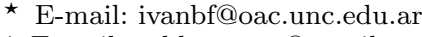

$\dagger$ E-mail: calderonoac@gmail.com
}

The Hipparcos mission (ESA 1997) for the first time provided high quality 5-parameters astrometric data (celestial coordinates, proper motion and parallax) of individual stars that led to unprecedent accuracy in the determination of their distances and tangential space velocities. Although it was limited to about 120000 stars in the solar neighbourhood, some near open clusters could be studied with great detail. The successor of Hipparcos, the Gaia mission (Gaia Collaboration et al. 2016) is making a significant improvement in the quality and quantity of astrometric and photometric data for individual stars. The Second Gaia Data Release (hereafter Gaia DR2) Gaia Collaboration et al. (2018a) allows detailed studies of the properties of galactic and extragalactic stellar systems, e.g. Gaia Collaboration et al. (2018b), Cantat-Gaudin et al. (2018a), Cantat-Gaudin et al. (2018b) and Soubiran et al. (2018).

In this work we develop and test a method to identify possible members of globular clusters from Gaia DR2 that consists of two stages: the first one is based on a clustering algorithm applied in a multi-dimensional space of physical parameters, for instance positions, proper motions and parallaxes. In most of the analysed clusters this stage alone cannot find all the members, so a second stage with a different approach is necessary in order to extract the members that have not been found up to this point. This second stage is 
Table 1. ICRS coordinates and galactic coordinates of the selected clusters.

\begin{tabular}{lcccc}
\hline Cluster & $\begin{array}{c}\text { RA } \\
\text { (HH MM SS.SS) }\end{array}$ & $\begin{array}{c}\text { DE } \\
\text { (DD MM SS.S) }\end{array}$ & $\begin{array}{c}l \\
(\mathrm{deg})\end{array}$ & $\begin{array}{c}b \\
(\mathrm{deg})\end{array}$ \\
\hline NGC 1261 & 03 12 16.21 & -551258.4 & 270.53871 & -52.12435 \\
NGC 3201 & 101736.82 & -462444.9 & 277.22878 & +08.64038 \\
NGC 6139 & 162739.99 & -385057.0 & 342.36464 & +06.93954 \\
NGC 6205 & 164141.63 & +362740.8 & 059.00947 & +40.91176 \\
NGC 6362 & 173154.99 & -670254.0 & 325.55452 & -17.56977 \\
NGC 6397 & 174042.09 & -534027.6 & 338.16501 & -11.95952 \\
NGC 6712 & 185304.32 & -084221.5 & 025.35410 & -04.31800 \\
Pal 13 & 230644.48 & +124619.2 & 087.10379 & -42.69993 \\
\hline
\end{tabular}

based on the analysis of the projected spatial distribution of stars with different proper motions around the mean proper motion of the cluster. Finally, we estimate the completeness and the degree of contamination, i.e. the fraction of expected members that were extracted and the number of field stars that could have been erroneously labelled as members, respectively.

We selected the clusters in this work by the fact that we have worked, or we are working on them. Namely NGC 3201 (Arellano Ferro et al. 2014), NGC 6362 (Arellano Ferro et al. 2018), NGC 6205 (Deras et al. 2019), NGC 6397, NGC 6139, NGC 1261, NGC 6712 and Palomar 13 (Yepez et al. 2019). Their coordinates are shown in Table 1.

\section{METHODOLOGY}

The extraction of the members of each cluster was performed in two stages. In this section we describe them in some detail.

\subsection{First stage}

The aim of this stage is to found groups of stars with similar characteristics in positions and/or motions. It was developed testing the different codes existing in Python for all the clustering algorithms provided by Scikit-Learn (Pedregosa et al. 2011), namely: K-means, affinity propagation, mean-shift, spectral clustering, hierarchical clustering, DBSCAN, gaussian mixtures and BIRCH. Each clustering algorithm was tested on different multidimensional spaces involving celestial coordinates $(\alpha, \delta)$ or their gnomonic projection $\left(X_{t}, Y_{t}\right)$, proper motions $\left(\mu_{\alpha *}=\mu_{\alpha} \cos \delta, \mu_{\delta}\right)$ or tangential velocities $\left(V t_{\alpha}, V t_{\delta}\right)$, and parallaxes $(\varpi)$ or distances $(R)$ as well as rectangular barycentric coordinates $(X, Y, Z)$. For instance in two dimensions: $\left[\mu_{\alpha *}, \mu_{\delta}\right]$; in three dimensions: $\left[\mu_{\alpha *}, \mu_{\delta}, \varpi\right]$ and $\left[V t_{\alpha}, V t_{\delta}, R\right]$; in four dimensions: $\left[\alpha, \delta, \mu_{\alpha *}, \mu_{\delta}\right],\left[X_{t}, Y_{t}, V t_{\alpha}, V t_{\delta}\right]$ and $\left[X_{t}, Y_{t}, \mu_{\alpha *}, \mu_{\delta}\right]$; in five dimensions: $\left[\alpha, \delta, \mu_{\alpha *}, \mu_{\delta}, \varpi\right], \quad\left[\alpha, \delta, V t_{\alpha}, V t_{\delta}, \quad \mathrm{R}\right],[$ $\left.X, Y, Z, V t_{\alpha}, V t_{\delta}\right],\left[X_{t}, Y_{t}, R, V t_{\alpha}, V t_{\delta}\right]$.

The initial tries were performed on the fields of wellknown near open and globular clusters. After these tries we noticed that when using parallaxes data, their relatively large uncertainty increased the dispersion of the entry data, consequently reducing the efficiency of the clustering algorithms. Therefore, we decided not to use parallaxes nor parameters computed from them, namely distances, rectangular barycentric coordinates and tangential velocities. Furthermore, since the geometry of spherical coordinates causes that clusters at different declinations (or latitudes when working in galactic coordinates) be treated in different ways, we chose to use tangential plane coordinates $\left(X_{t}, Y_{t}\right)$ instead of celestial coordinates. We finally decided to run the clustering algorithms in the four-dimensional space $\left[X_{t}, Y_{t}, \mu_{\alpha *}, \mu_{\delta}\right]$. This first stage consists of several steps that are briefly summarized here:

- Download Gaia DR2 in a cone around the cluster with radius around two tidal radii from Kharchenko et al. (2013) and compute the gnomonic projection of the celestial coordinates: $\left(X_{t}, Y_{t}\right)$ (Astropy Collaboration et al. 2013, 2018).

- Pre-process the data $\left(X_{t}, Y_{t}, \mu_{\alpha *}, \mu_{\delta}\right)$ using RobustScaler in Scikit-Learn, in order to remove outliers and normalize the different dimensions. This normalization is necessary because the very different range of the values of coordinates and proper motions.

- Run the BIRCH clustering algorithm (Zhang, Ramakrishnan \& Livny 1996) in the 4D space: $\left[X_{t}, Y_{t}, \mu_{\alpha *}, \mu_{\delta}\right]$.

- Fit a $4 \mathrm{D}$ gaussian distribution with the candidate members of every cluster identified by the algorithm.

- Discard candidate members that lie outside the $3 \sigma$ ellipsoid and fit a new 4D gaussian distribution.

- Retain as candidate members of the cluster only the stars within the $3 \sigma$ ellipsoid of the last fitted gaussian distribution.

- Plot the space distribution, the vector point diagram -hereafter VPD- and the color magnitude diagram in Gaia DR2 photometric system (Gmag, BPmag - RPmag) hereafter $\mathrm{CMD}$ - with the possible members of the clusters found.

- Verify that the space distribution, the VPD and the CMD are consistent with those of a stellar cluster. This is the only step in this stage that is still performed by a human being.

The results of the first stage of the membership determination on six globular clusters are displayed in Figure 1. The candidate member stars of all the clusters in Figure 1 show: projected spatial positions that correspond to a spherical distribution, proper motions that are very concentrated as expected in the VPD of a globular cluster, and a CMD with the characteristic features of a globular cluster, e.g. the turn-off, the red giant branch and the horizontal branch. As can be noticed from the VPDs in Figure 1, there are different situations regarding the distribution of the proper motions of the cluster members relative to that of the field stars: the proper motions of the members of NGC 3201 and NGC 6397 are clearly separated from the proper motions of the field stars, while in the remaining clusters both distributions are overlapped. Furthermore, in NGC 1261 and NGC 6205 the mean proper motion of the cluster is very close to the mean proper motion of the field stars.

This first stage was employed by Deras et al. (2019) to find the most likely members of the globular cluster NGC 6205 in order to build a clean CMD and to confirm the membership status of all known variable stars.

It is well known from studies on the density profiles of globular clusters (de Boer et al. 2019) that they do not have definite borders, and diffuse stellar envelopes have been found surrounding some of them (Kuzma et al. 2018). However, it can be seen in Figure 1 that after the application of this stage, sharply defined borders are observed in the fields and in the VPDs. It must be noticed that they do not 
reflect physical features of the clusters, since they are a consequence of the $3 \sigma$ cut imposed to the ellipsoid in the $4 \mathrm{D}$ space $\left[X_{t}, Y_{t}, \mu_{\alpha *}, \mu_{\delta}\right]$. Therefore, there are members outside those artificial borders that will be extracted in the second stage with a different approach not based on any clustering algorithm.

\subsection{Second stage}

Any method for the determination of the members of a cluster could include some field star as members or vice versa. In order to estimate the number of missing members we computed the radial density projected on the sky of all the stars in the field with measured proper motions. As an example, in Figure 2 we show the radial density profile in the field of radius $40^{\prime}$ around the centre of NGC 6205 that contains 40083 stars with measured proper motions. Here the background density was estimated to be $1.8 \mathrm{stars} / \mathrm{arcmin}^{2}$, therefore the studied field is expected to have 9000 field stars and 31000 members of the cluster, however the first stage extracts only 23070 possible members, a number significantly less than expected. Table 2 summarizes the results of similar analysis performed in all the fields studied in this work and the number of members extracted in the first stage.

It is expected that field stars show a uniform spatial distribution, but instead in most of the fields we found that the stars not identified as members in the first stage show a residual overdensity in the same position of the cluster, and also a residual concentration in the VPD around the mean proper motion of the members (see Figure 3 and examples in Appendix A). Notice that the stars corresponding to the peak at the mean proper motion of the members detected in the first stage lie at the outskirts of the cluster and most of them are very likely members. Actually, the stars clustered in proper motions are spread in space, while the ones clustered in spatial positions are spread in proper motions, therefore in at least one of the four dimensions they lie outside the $3 \sigma$ ellipsoid in the $4 \mathrm{D}$ space. That is why the first stage does not detect them.

These observations and the results in Table 2 show that there is a significant number of members that were not detected in the first stage, most of which are expected to be found in this second stage. Only in the case of Pal 13, after the first stage there are no residual overdensities neither in the field nor in the VPD, therefore the second stage was not performed.

For a better understanding of this second stage it is necessary to describe in some detail the morphological characteristics of the distribution in the VPD of the stars that were not detected as members in the first stage around the mean proper motion of the cluster. As can be seen in the lower panel of Figure 3, it consists of a symmetrical radial distribution with a noticeable central peak without a definite border that is surrounded by a low-density circular area that finishes in a sharp border, after which it decays smoothly outwards. The observed symmetry in this residual distribution suggests that the best way analyse it is by means of rings of increasing radii centred in the mean proper motion of the cluster. It is remarkable to notice that this feature in VPD appears in all the analysed clusters except Pal 13, consequently one may be led to conclude that it is not related to a physical property of the cluster but a consequence of the extraction of members with the first stage of the method.

The second stage also consists of several steps that are briefly summarized here:

- Select rings of proper motions centred in the mean proper motion of the detected members, with increasing radii.

- Compute the radial density projected on the sky of all the stars within each ring of proper motions.

- Determine for every radial density profile the mean density of the background and the radius of the central overdensity.

- From the stars that were not classified as members in the first stage, extract as new members those that lie within each ring of proper motions in the VPD and inside the corresponding central overdensity in the field.

The outer part of all the radial density profiles is approximately constant within their errors computed assuming Poisson noise in the counts. That constant density was assumed as the background density and it was estimated as the average of the density values in that outer part.

For a better description of this stage, in Appendix A we show the application on two examples representing the two different situations mentioned above regarding the proper motions: NGC 1261, where the distribution of the proper motions of the cluster and of the field stars are overlapped, and NGC 3201, where both distributions are well separated. Figure 4 shows all the members of NGC 1261 and NGC 3201 that were extracted after the two stages.

\section{RESULTS}

In Table 3 we show the number of members we found in Gaia DR2 for every cluster, their mean proper motions, and the radius from the cluster centre that contains all the detected members $\left(R_{\max }\right)$. In this work the mean proper motion of every cluster was computed as the simple average of the individual proper motions of the determined member stars, without any consideration about their errors and covariances. The uncertainties on the mean proper motions were computed considering the contributions due to random and systematic errors. The last ones were estimated using the spatial covariance of proper motions errors derived by Lindegren et al. (2018).

For comparison we show the results of Gaia Collaboration et al. (2018b) -GC18 hereafter-, Baumgardt et al. (2019) -BHSB19 hereafter- and Vasiliev (2019) -Vas19 hereafterThe uncertainties in our work are similar to those in Vas19 since they were calculated following an approach alike, whereas GC18 and BHSB19 do not take systematic errors into account and consequently their formal errors are smaller. Note that some clusters were not analysed by GC18, since their sample is mostly formed by clusters closer than $12 \mathrm{kpc}$.

Table 4 shows some rows of the table containing all the sources in Gaia DR2 in the studied fields with their membership status. The full table is available online. 
Table 2. Estimation of the number of members of every cluster based on the radial density profiles of stars with measured proper motion. $\left(^{*}\right)$ The background density is so high that the uncertainty in the number of field stars is larger than the estimated number of cluster members. $\left(^{* *}\right)$ It is a very poor cluster, so the uncertainty in the number of field stars is larger than the estimated number of cluster members.

\begin{tabular}{|c|c|c|c|c|c|c|c|}
\hline Cluster & $\begin{array}{l}\text { Field radius } \\
\text { (arcmin) }\end{array}$ & $\begin{array}{l}\text { Gaia DR2 } \\
\text { stars }\end{array}$ & $\begin{array}{c}\text { Stars with } \\
\text { proper motion }\end{array}$ & $\begin{array}{c}\text { Background } \\
\text { density }\left(\operatorname{arcmin}^{-2}\right)\end{array}$ & $\begin{array}{l}\text { Expected } \\
\text { field stars }\end{array}$ & $\begin{array}{l}\text { Expected cluster } \\
\text { members with PM }\end{array}$ & $\begin{array}{l}\text { Members extracted } \\
\text { in first stage }\end{array}$ \\
\hline NGC 1261 & 20 & 9448 & 6558 & $1.02 \pm 0.15$ & $1280 \pm 190$ & $5300 \pm 190$ & 3024 \\
\hline NGC 3201 & 60 & 229979 & 207158 & $15.77 \pm 0.35$ & $178400 \pm 3900$ & $28800 \pm 3900$ & 22200 \\
\hline NGC 6139 & 20 & 69729 & 45384 & $34.42 \pm 1.04$ & $43300 \pm 1300$ & $2100 \pm 1300$ & 1462 \\
\hline NGC 6205 & 40 & 52802 & 40083 & $1.82 \pm 0.15$ & $9150 \pm 750$ & $30900 \pm 750$ & 23070 \\
\hline NGC 6362 & 25 & 54706 & 43896 & $14.32 \pm 0.58$ & $28100 \pm 1100$ & $15800 \pm 1100$ & 12217 \\
\hline NGC 6397 & 60 & 472304 & 383900 & $31.55 \pm 0.53$ & $35700 \pm 6000$ & $27100 \pm 6000$ & 21997 \\
\hline NGC 6712 & 10 & 60447 & 34231 & $106.20 \pm 3.52$ & $33400 \pm 1100$ & $900 \pm 1100(*)$ & 982 \\
\hline Pal 13 & 10 & 457 & 399 & $1.07 \pm 0.27$ & $340 \pm 90$ & $60 \pm 90(* *)$ & 69 \\
\hline
\end{tabular}

Table 3. Number of members extracted in every cluster and their mean proper motions with their uncertainties, in this work, in GC18, in BHSB19 and in Vas19. All the proper motions are expressed in mas/yr and $R_{\max }$ in arcmin.

\begin{tabular}{|c|c|c|c|c|c|c|c|c|c|c|c|c|c|}
\hline \multirow[t]{3}{*}{ Cluster } & \multicolumn{4}{|c|}{ This work } & \multicolumn{4}{|c|}{ CG18 } & \multicolumn{2}{|c|}{ BHSB19 } & \multicolumn{3}{|c|}{ Vas19 } \\
\hline & Members & $\mu_{\alpha *}$ & $\mu_{\delta}$ & $R_{\max }$ & Members & $\mu_{\alpha *}$ & $\mu_{\delta}$ & $R_{\max }$ & $\mu_{\alpha *}$ & $\mu_{\delta}$ & Members & $\mu_{\alpha *}$ & $\mu_{\delta}$ \\
\hline & & $\epsilon_{\mu_{\alpha *}}$ & $\epsilon_{\mu_{\delta}}$ & & & $\epsilon_{\mu_{\alpha *}}$ & $\epsilon_{\mu_{\delta}}$ & & $\epsilon_{\mu_{\alpha *}}$ & $\epsilon_{\mu_{\delta}}$ & & $\epsilon_{\mu_{\alpha *}}$ & $\epsilon_{\mu_{\delta}}$ \\
\hline \multirow[t]{2}{*}{ NGC 1261} & 5258 & 1.669 & -2.048 & 15.0 & - & - & - & - & 1.61 & -2.05 & 541 & 1.632 & -2.038 \\
\hline & & 0.069 & 0.068 & & & - & - & & 0.02 & 0.02 & & 0.057 & 0.057 \\
\hline \multirow[t]{2}{*}{ NGC 3201} & 31536 & 8.298 & -1.947 & 45.0 & 19921 & 8.3344 & -1.9895 & 58.8 & 8.35 & -2.00 & 7021 & 8.324 & -1.991 \\
\hline & & 0.043 & 0.043 & & & 0.0021 & 0.0020 & & 0.01 & 0.01 & & 0.044 & 0.044 \\
\hline \multirow[t]{2}{*}{ NGC 6139} & 2072 & -5.952 & -2.665 & 7.5 & - & - & - & - & -6.16 & -2.67 & 426 & -6.184 & -2.648 \\
\hline & & 0.075 & 0.069 & & & - & - & & 0.03 & 0.02 & & 0.062 & 0.061 \\
\hline \multirow[t]{2}{*}{ NGC 6205} & 31033 & -3.176 & -2.597 & 25.0 & 15634 & -3.1762 & -2.5876 & 34.8 & -3.18 & -2.56 & 3982 & -3.164 & -2.588 \\
\hline & & 0.051 & 0.052 & & & 0.0027 & 0.0030 & & 0.01 & 0.01 & & 0.047 & 0.047 \\
\hline \multirow[t]{2}{*}{ NGC 6362} & 16149 & -5.409 & -4.767 & 11.5 & 9169 & -5.5014 & -4.7417 & 15.6 & -5.49 & -4.76 & 2877 & -5.510 & -4.750 \\
\hline & & 0.062 & 0.062 & & & 0.0028 & 0.0032 & & 0.01 & 0.01 & & 0.051 & 0.052 \\
\hline \multirow[t]{2}{*}{ NGC 6397} & 30526 & 3.280 & -17.573 & 33.0 & 22116 & 3.2908 & -17.5908 & 45.6 & 3.30 & -17.60 & 11406 & 3.285 & -17.621 \\
\hline & & 0.039 & 0.039 & & & 0.0026 & 0.0025 & & 0.01 & 0.01 & & 0.043 & 0.043 \\
\hline \multirow[t]{2}{*}{ NGC 6712} & 1529 & 3.306 & -4.393 & 4.2 & - & - & - & - & 3.32 & -4.38 & 309 & 3.341 & -4.384 \\
\hline & & 0.068 & 0.069 & & & - & - & & 0.01 & 0.01 & & 0.062 & 0.061 \\
\hline \multirow[t]{2}{*}{ Pal 13} & 69 & 1.805 & 0.134 & 2.8 & - & - & - & - & 1.64 & 0.25 & 34 & 1.615 & 0.142 \\
\hline & & 0.341 & 0.211 & & & - & - & & 0.09 & 0.07 & & 0.101 & 0.089 \\
\hline
\end{tabular}

Table 4. Sample of the table available online with the membership status of all the Gaia DR2 sources in the studied fields. Column 1: Cluster in the centre of the field; column 2: Gaia DR2 source identifier; columns 3 to 7: coordinates, proper motions and G magnitude from Gaia DR2; column 8: membership status: M1 and M2 cluster member extracted in stage 1 or 2 respectively, FS field star, UN unknown status due to proper motion not available in Gaia DR2.

\begin{tabular}{cccccccc}
\hline Field & Source & $\begin{array}{c}\text { RA (ICRS) } \\
(\text { deg.) }\end{array}$ & $\begin{array}{c}\text { DE (ICRS) } \\
\text { (deg.) }\end{array}$ & $\begin{array}{c}\mu_{\alpha *} \\
\text { mas/yr }\end{array}$ & $\begin{array}{c}\mu_{\delta} \\
\text { mas/yr }\end{array}$ & Gmag & $\begin{array}{c}\text { Membership } \\
\text { status }\end{array}$ \\
\hline NGC6362 & 5813078054348813952 & 263.21601570027 & -67.14175729371 & -4.504 & -1.01 & 19.9794 & FS \\
NGC6362 & 5813078054348817280 & 263.21665026925 & -67.13299247263 & -5.541 & -4.891 & 17.8539 & M1 \\
NGC6362 & 5813078084397967104 & 263.24072593825 & -67.13770768894 & 0.811 & -3.248 & 18.9709 & FS \\
NGC6362 & 5813078084397982336 & 263.22608925444 & -67.13456042868 & -3.879 & -5.396 & 18.1761 & M2 \\
NGC6362 & 5813078084397982464 & 263.22985041340 & -67.13388363600 & -5.752 & -5.313 & 19.0014 & M1 \\
NGC6362 & 5813078084397983744 & 263.24810934919 & -67.13319244657 & & & 21.3399 & UN \\
\hline
\end{tabular}

Table 5. Estimation of the completeness of the extracted members.

\begin{tabular}{lccccccc}
\hline Cluster & $\begin{array}{c}\text { Field radius } \\
\text { (arcmin) }\end{array}$ & $\begin{array}{c}\text { Gaia DR2 } \\
\text { stars }\end{array}$ & $\begin{array}{c}\text { Background } \\
\text { density }\left(\text { arcmin }^{-2}\right)\end{array}$ & $\begin{array}{c}\text { Expected } \\
\text { field stars }\end{array}$ & $\begin{array}{c}\text { Expected cluster } \\
\text { members }\end{array}$ & $\begin{array}{c}\text { Total members } \\
\text { extracted }\end{array}$ & $\begin{array}{c}\% \text { Total } \\
\text { members }\end{array}$ \\
\hline NGC 1261 & 20 & 9448 & 1.2 & 1508 & 7940 & 5258 & 66 \\
NGC 3201 & 60 & 229979 & 17.0 & 192265 & 37714 & 31536 & 84 \\
NGC 6139 & 20 & 69729 & 51.0 & 64088 & 5641 & 2072 & 37 \\
NGC 6205 & 40 & 52802 & 2.0 & 10053 & 42749 & 31033 & 73 \\
NGC 6362 & 25 & 54706 & 16.8 & 32987 & 21719 & 16149 & 74 \\
NGC 6397 & 60 & 472304 & 38.3 & 433163 & 39141 & 30526 & 78 \\
NGC 6712 & 10 & 60447 & 180.0 & 56549 & 3898 & 1529 & 39 \\
Pal 13 & 10 & 457 & 1.2 & 380 & 77 & 69 & 90 \\
\hline
\end{tabular}



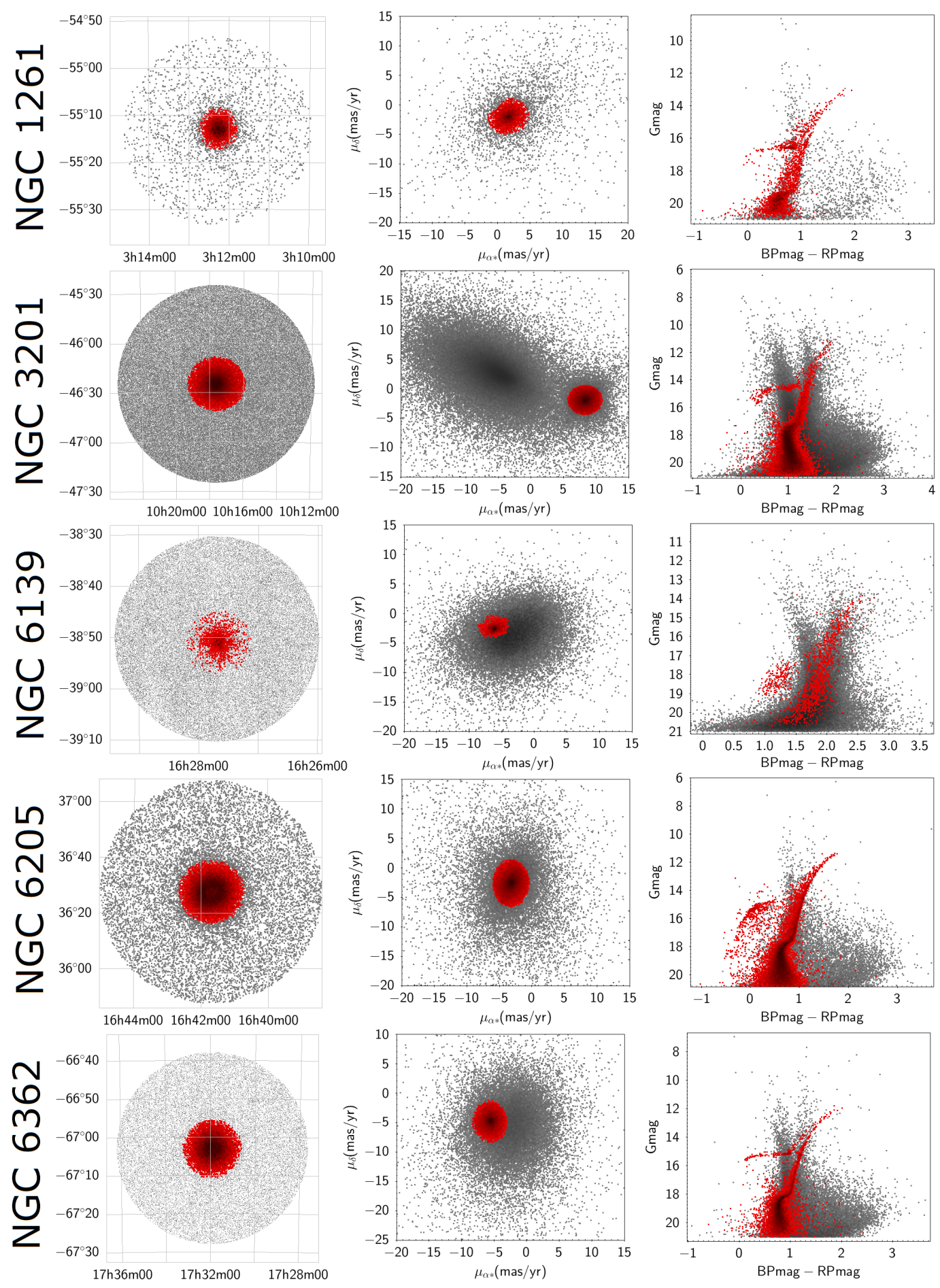

Figure 1. Members extracted in the first stage (red) and remaining Gaia DR2 sample (gray) in the field of the selected clusters. Left: position in the sky, ICRS coordinates; middle: VPD; right: CMD. Notice that, as mentioned in Section 2.1, the first stage only extracts a fraction of the possible members.

\section{EFFICIENCY OF THE EXTRACTION}

In order to analyse the efficiency of the extraction method we perform an estimation of the completeness -fraction of the total number of expected members that is detected- and the degree of contamination of the extracted members - number of field stars that could have been labelled as members of the cluster-.
The developed method relies on the proper motions for the determination of the membership status of every star but there are many stars in Gaia DR2 with no measured proper motions, therefore several cluster members cannot be extracted. By assuming a constant projected background density, and that all the stars above that background are members of the cluster, it is possible to estimate the total 

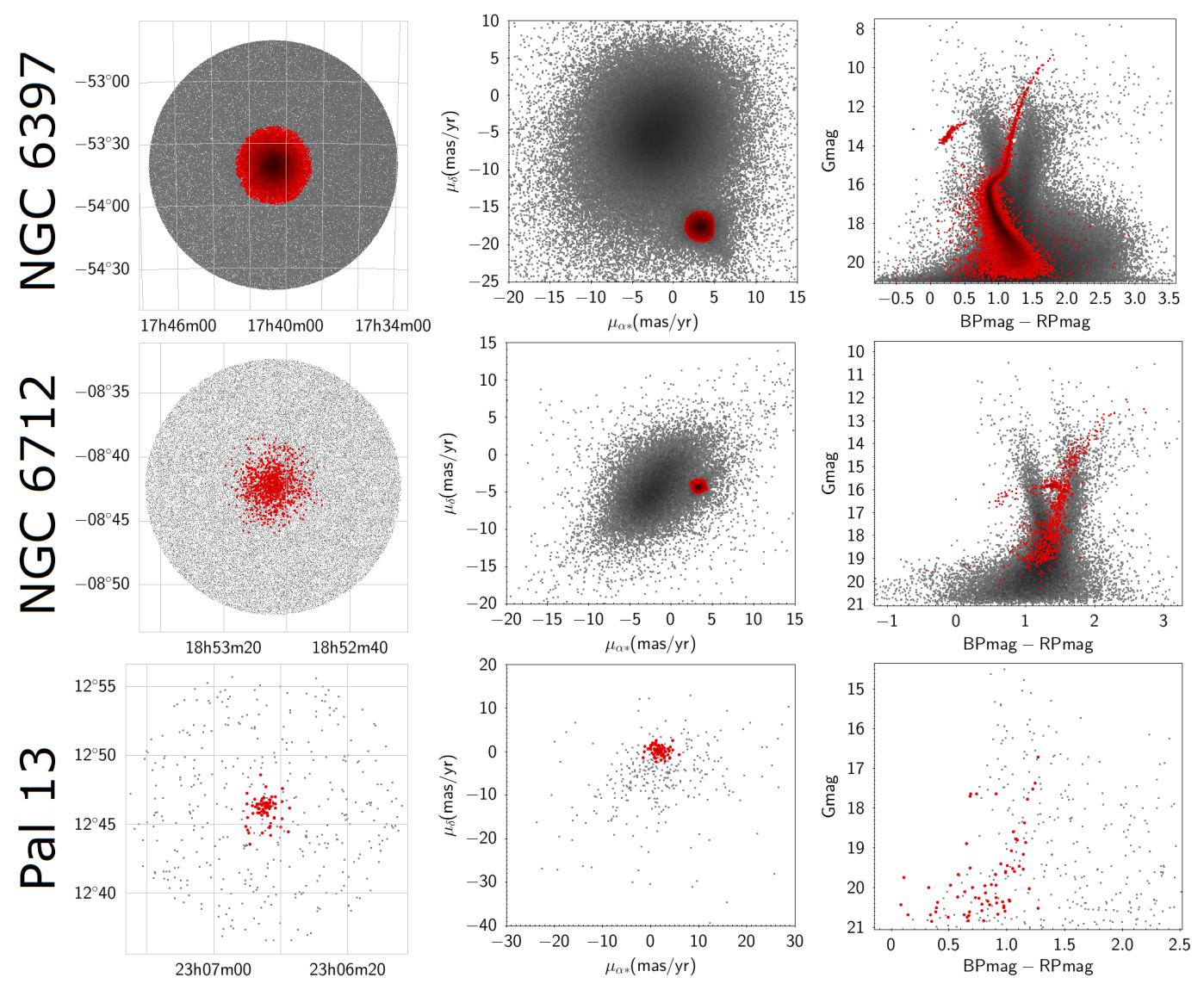

Figure 1 - continued Members extracted in the first stage (red) and remaining Gaia DR2 sample (gray) in the field of the selected clusters. Left: position in the sky, ICRS coordinates; middle: VPD; right: CMD.

Table 6. Estimation of the contamination of the extracted members.

\begin{tabular}{lccccccccc}
\hline Cluster & $\begin{array}{c}\text { Members } \\
\text { 1st stage }\end{array}$ & $\begin{array}{c}\text { Field stars } \\
\text { 1st stage }\end{array}$ & $\begin{array}{c}\text { Contamination } \\
\text { 1st stage (\%) }\end{array}$ & $\begin{array}{c}\text { Members } \\
\text { 2nd stage }\end{array}$ & $\begin{array}{c}\text { Field stars } \\
\text { 2nd stage }\end{array}$ & $\begin{array}{c}\text { Contamination } \\
\text { 2nd stage (\%) }\end{array}$ & $\begin{array}{c}\text { Total } \\
\text { members }\end{array}$ & $\begin{array}{c}\text { Total } \\
\text { field stars }\end{array}$ & $\begin{array}{c}\text { Overall } \\
\text { contamination (\%) }\end{array}$ \\
\hline NGC 1261 & 3024 & 32 & 1 & 2234 & 100 & 4 & 5258 & 132 & 3 \\
NGC 3201 & 22200 & 3 & 0 & 9336 & 348 & 4 & 31536 & 385 & 1 \\
NGC 6139 & 1462 & 297 & 20 & 610 & 461 & 76 & 2072 & 758 & 37 \\
NGC 6205 & 23070 & 181 & 1 & 7963 & 543 & 7 & 31033 & 724 & 2 \\
NGC 6362 & 12217 & 209 & 2 & 3932 & 982 & 25 & 16149 & 1191 & 7 \\
NGC 6397 & 21997 & 89 & 0 & 8529 & 569 & 7 & 30526 & 658 & 2 \\
NGC 6712 & 982 & 169 & 17 & 547 & 107 & 20 & 1529 & 276 & 3 \\
Pal 13 & 69 & 3 & 4 & - & - & - & 69 & 3 \\
\hline
\end{tabular}

number of members that could be found in Gaia DR2, despite their proper motions could have not been measured. Arenou et al. (2018) discuss the completeness of the Gaia DR2 catalog and they show that in very crowded regions the completeness level depends on both magnitude and local density. In the centre of globular clusters they found completeness below $75 \%$ at Gmag20, and specifically in the centre of NGC 1261 and NGC 6205 almost all the stars with Gmag > 19 are missing in Gaia DR2.

Performing an analysis like that mentioned in the beginning of Section 2.2 but counting all the stars in the field instead of only those with measured proper motion, we obtain the estimations in Table 5. It must be noticed that the percentages in the last column are upper limits to the actual completeness for the most favourable cases (low density fields), since the incompleteness of the Gaia DR2 catalog itself has not been taken into account in their computations.

On the other hand, since the distributions of field stars and of cluster members are overlapped both in the sky and in proper motions, it is also expected that some field stars could have been erroneously extracted as members. Those stars are what we call contamination of the members. Table 6 shows as field stars (columns 3 and 6) the number of field stars that are expected to be located in the same region of the sky and of the VPD that the extracted members, therefore they could have been erroneously labelled as members. The clusters more affected by contamination are those in the regions with the highest densities of field stars. Note also that in all cases the set of members extracted in the first stage is less affected by contamination than those extracted in the second stage. 

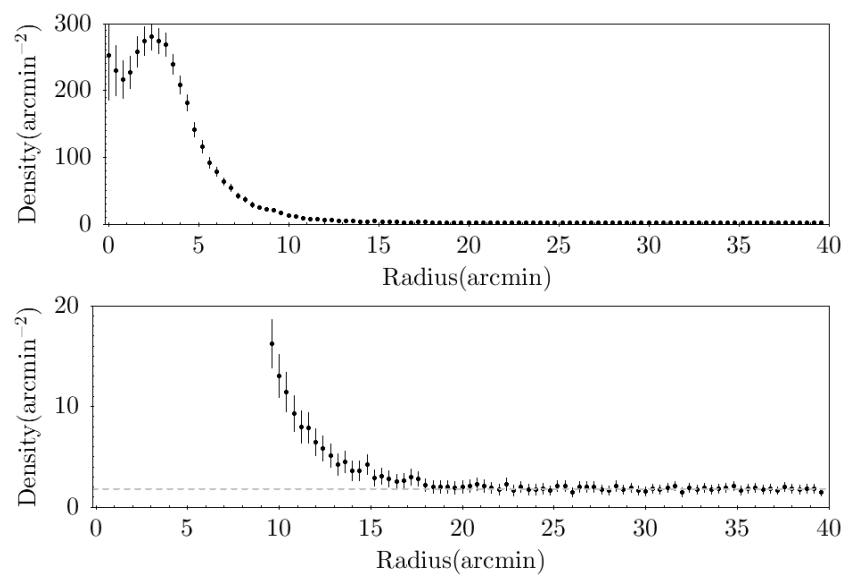

Figure 2. Upper panel: Radial density projected on the sky of all the stars with measured proper motions in the field of radius $40^{\prime}$ around NGC 6205, with $3 \sigma$ error bars computed assuming Poisson noise in the counts $(*)$. Lower panel: the same as upper panel with expanded vertical scale, and horizontal gray dotted line showing the adopted background density. (*) The drop in the central region of the cluster is due to the incompleteness of Gaia DR2 proper motions in very crowded fields.

\section{CONCLUSIONS}

The method developed was able to extract the members of eight globular clusters in a variety of backgrounds from a sample of Gaia DR2 stars with proper motions. A statistical analysis of members and field stars shows that between $37 \%$ and $90 \%$ of the expected number of members were extracted, but the number of members not extracted is mostly due to the lack of proper motions in a large fraction of stars. In the fields studied in this work, between $10 \%$ and $43 \%$ of the stars do not have their proper motions measured in Gaia DR2.

The mean proper motions of the clusters measured with our method are in excellent agreement with those found by other authors that make use of the same input catalogue (Gaia DR2). In addition to that our method extracted between $38 \%$ and $98 \%$ more members than GC18.

The contamination of the cluster members by field stars was evaluated. In six of the eight studied clusters it was estimated to be between $1 \%$ and $7 \%$. Only NGC 6139 and NGC 6712 present significantly larger estimated contaminations, presumably due to the overlap of the proper motions distributions corresponding to the cluster and the field, and the very crowded backgrounds with the highest average projected stellar densities in this study: 51 and 180 stars per $\operatorname{arcmin}^{2}$ respectively.

The members found by means of the method presented here have been used to successfully clean the colourmagnitude diagrams of the globular clusters NGC 6205 and Pal 13, in order to derive more reliable astrophysical parameters. This method has also been used to confirm the membership of variable stars in NGC 3201, NGC 6205, NGC 6362 and Pal 13 (see references in Section 1).
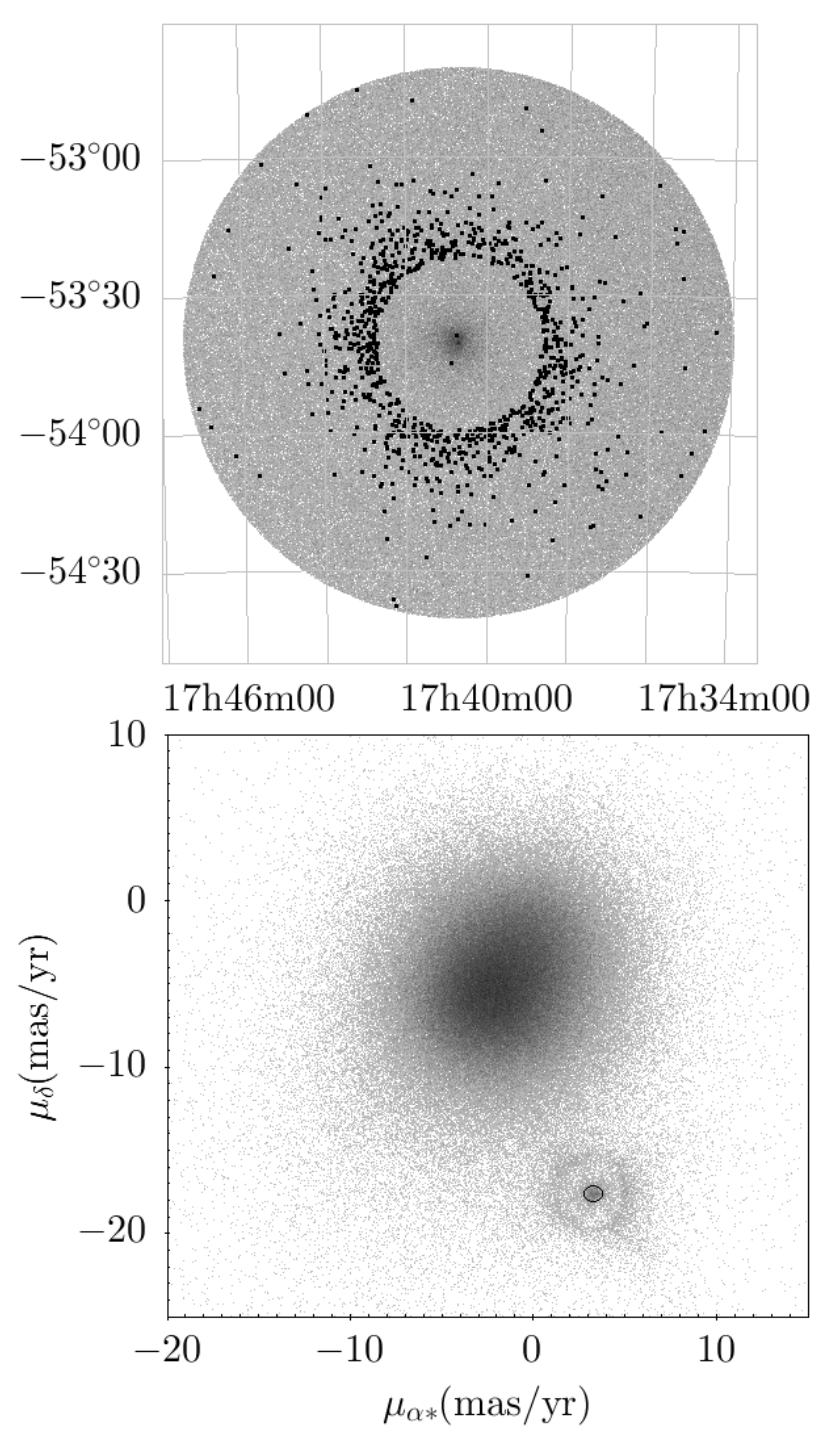

Figure 3. Positions on the field (upper panel) and in the VPD (lower panel) of the stars that were not detected as members of NGC 6397 in the first stage. The stars marked with black dots in the field are the ones enclosed in the circle of radius $0.5 \mathrm{mas} / \mathrm{yr}$ around the mean proper motion of the cluster members in the VPD.

\section{ACKNOWLEDGEMENTS}

This research has made use of the SIMBAD database, operated at CDS, Strasbourg, France (Wenger et al. 2000). This research has also made use of the software TOPCAT (Taylor 2005).

\section{REFERENCES}

Arellano Ferro A., Ahumada J.A., Bustos Fierro I.H., Calderón J.H., Morrell N.I., 2018, Astronomische Nachrichten, Vol. 339, Issue 183 , pp. 183-197

Arellano Ferro A., Ahumada J.A., Calderón J.H., Kains N., 2014, Rev. Mex. Astron. Astrofis., Vol. 50, pp. 307-322

Arenou, F., Luri, X., Babusiaux, C. et al. 2018, A\&A, Vol. 616, id.A17, 29pp. 

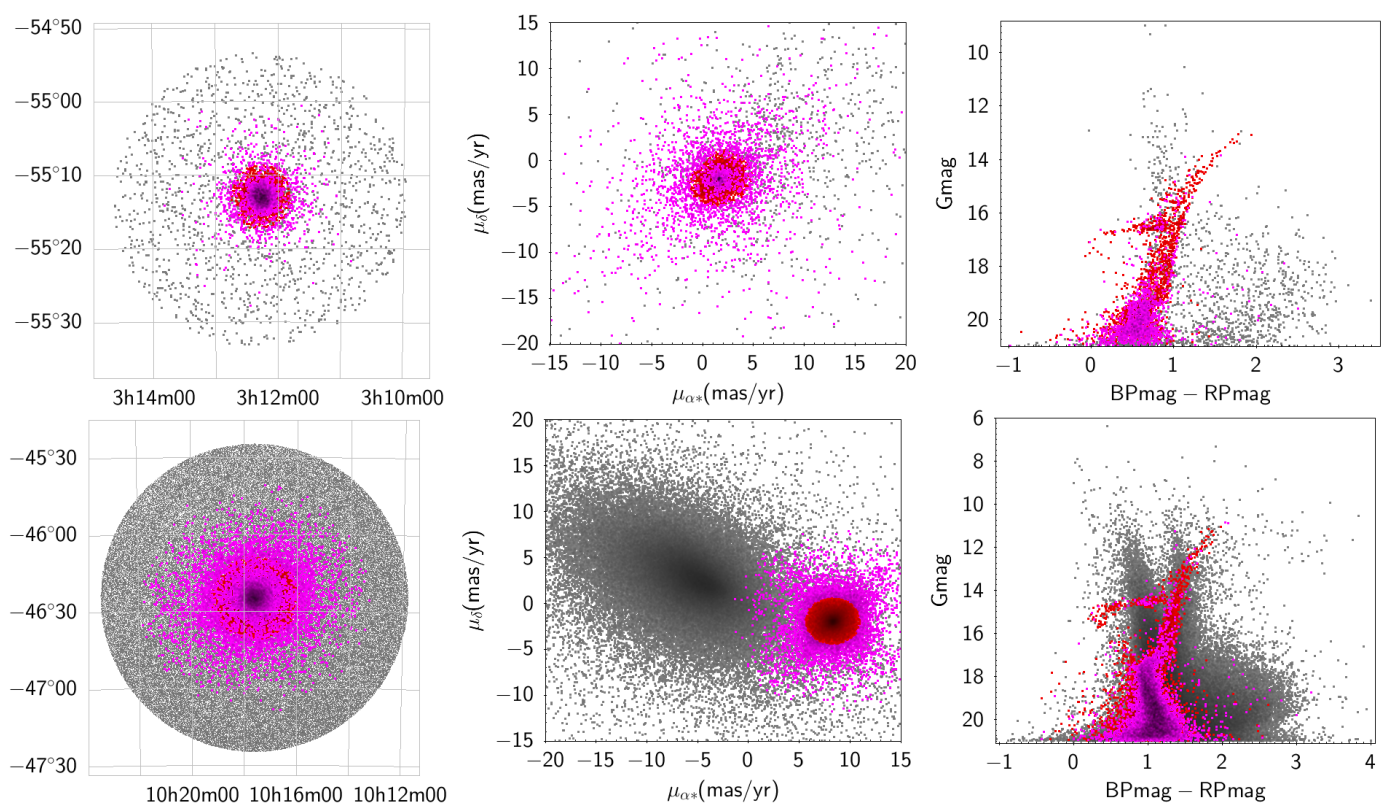

Figure 4. Members extracted in the first stage (red), in the second stage (pink) and remaining Gaia DR2 sample (gray) in the field of NGC 1261 (upper panel) and NGC 3201 (lower panel). Left: position in the sky, ICRS coordinates; middle: VPD; right: CMD. Note: In all the graphics the pink dots are plotted over the red dots, except in the VPD of NGC 3201 where the pink dots are plotted under the red dots.

Astropy Collaboration 2013, A\&A, Vol. 558, id. A33, 9 pp.

Astropy Collaboration 2018, AJ, Vol. 156, Issue 3, id. 123, 19 pp.

Baumgardt H., Hilker M., Sollima A., Bellini A., 2019, MNRAS, Vol. 482, Issue 4, p.5138-5155

Cantat-Gaudin T., Jordi C., Vallenari A., et al. 2018a, A\&A, 618, A93

Cantat-Gaudin T., Krone-Martins A., Sedaghat N., et al. 2018b, ArXiv e-prints, arXiv:1810.05494

de Boer T.J.L., Gieles M., Balbinot E., HÃl'nault-Brunet V., Sollima A., Watkins L.L., Claydon I., 2019, MNRAS, Vol. 485, Issue 4, p.4906-4935

Deras D., Arellano Ferro A., Lázaro C., Bustos Fierro I.H., Calderón J.H., Muneer S., Giridhar S., 2019, A new study of the variable star population in the Hercules globular cluster (M13; NGC 6205), MNRAS, stz642, https://doi.org/10.1093/mnras/stz642

ESA 1997, The Hipparcos and Tycho catalogues. Astrometric and photometric star catalogues derived from the ESA Hipparcos Space Astrometry Mission, Publisher: Noordwijk, Netherlands: ESA Publications Division, 1997, Series: ESA SP Series vol no: 1200, ISBN: 9290923997

Gaia Collaboration, Prusti T., de Bruijne J.H.J., et al. 2016, A\&A, 595, A1

Gaia Collaboration, Brown A.G.A., Vallenari A., Prusti T., et al. 2018a, A\&A, Vol. 616, id. A1, 22pp

Gaia Collaboration, Helmi A., van Leeuwen F., et al. 2018b, A\&A, Vol. 616, id. A12, 47 pp

Galadí-Enríquez D., Jordi C., Trullols E., 1998, A\&A, Vol. 337, p.125-140

Kharchenko N.V., Piskunov A.E., Roeser S., Schilbach E., Scholz R.-D. 2013, A\&A, Vol. 558, A53

Kuzma P.B., Da Costa G.S., Mackey A.D., 2018, MNRAS, Vol. 473, Issue 3, p.2881-2898

Lindegren, L., Hernández, J., Bombrun, A., et al. 2018, A\&A, Vol. 616, id.A2, 25 pp.

Pedregosa et al., 2011, Scikit-learn: Machine Learning in Python, Journal of Machine Learning Research 12, p.2825-2830

Soubiran C., Cantat-Gaudin T., Romero-Gomez M., et al. 2018,
A\&A, 619, A155

Vasilevskis S., Sanders W.L., van Altena W.F., 1965, AJ, Vol. 70, p. 806

Vasiliev E., 2019, MNRAS, Vol. 484, Issue 2, p.2832-2850

Yepez M.A., Arellano Ferro A., Schröder K. P., Muneer S., Giridhar S., Allen C., 2019, New Astron., Vol. 71, p. 1-11

Zhang T., Ramakrishnan, R. and Livny, M., 1996, BIRCH: An Efficient Data Clustering Method for Very Large Databases. 1996 ACM SIGMOD International Conference on Management of Data, Canada, 4-6 June 1996, 103-114

\section{APPENDIX A: EXAMPLES}

This appendix illustrates the procedure of the second stage of members extraction by showing two examples. In the first one (NGC 1261) the distributions of proper motions of the cluster and of field stars are overlapped and their mean proper motions are similar. In the second one (NGC 3201) those distributions are clearly separated.

\section{A1 NGC 1261}

In Figure A1 we show the residual overdensity in the field and the residual concentration in the VPD around the mean proper motion of NGC 1261 after the first stage of members extraction. In this case the distribution of the proper motions of the cluster and of the field stars are overlapped, a different situation of that shown in Figure 3 where the distributions are clearly separated.

In the first stage we found 3024 members with mean proper motion $\mu_{\alpha *}=1.6756 \mathrm{mas} / y r, \mu_{\delta}=-2.0440 \mathrm{mas} / y r$. We selected the stars in Gaia DR2 in rings of proper motions centred in this mean proper motion with increasing radii as shown in Figure A2.

We computed the radial density projected on the sky of 

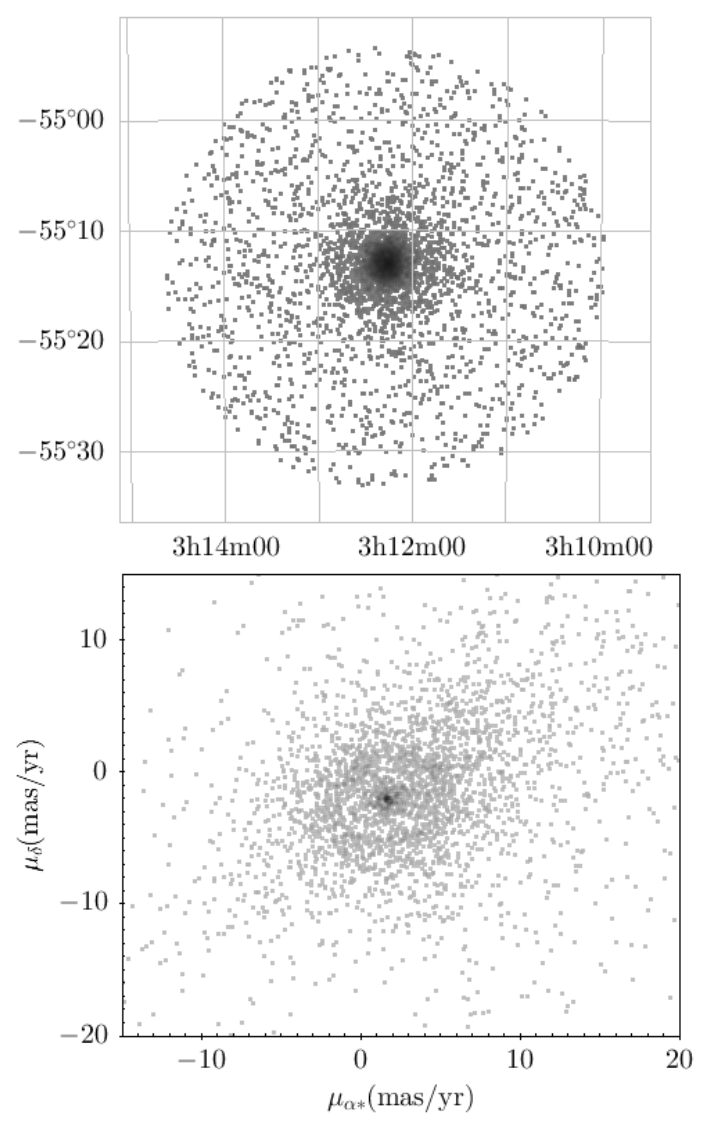

Figure A1. Positions on the field (upper panel) and in the VPD (lower panel) of the stars that were not detected as members of NGC 1261 in the first stage.

all the Gaia DR2 stars within each ring of proper motions, some of which are shown in Figure A3. It is important to notice that the stars in the innermost rings (proper motions very close to the mean proper motion of the cluster) fill a wider region on the field, while the stars in the outermost rings (larger differences in proper motions relative to the mean) are very concentrated in the central region of the cluster. This fact reflects the high velocity dispersion in that region.

From the list of stars that were not classified as members in the first stage, we select those inside each ring of proper motions that in the field are lying within the radius of the central overdensity corresponding to that ring, and we add them to the list of members of the cluster. As result of this stage 2234 more members were extracted. The distribution of the resulting field stars is shown in Figure A4, where there is no residual overdensity in the field nor residual concentration in the VPD, and the CMD does not show the typical features of a globular cluster.

Other way of verifying that the number of missing members is neglectable is by performing radial counts of the field stars that increases linearly with the radius if they have a constant density, as can be seen in Figure A5.

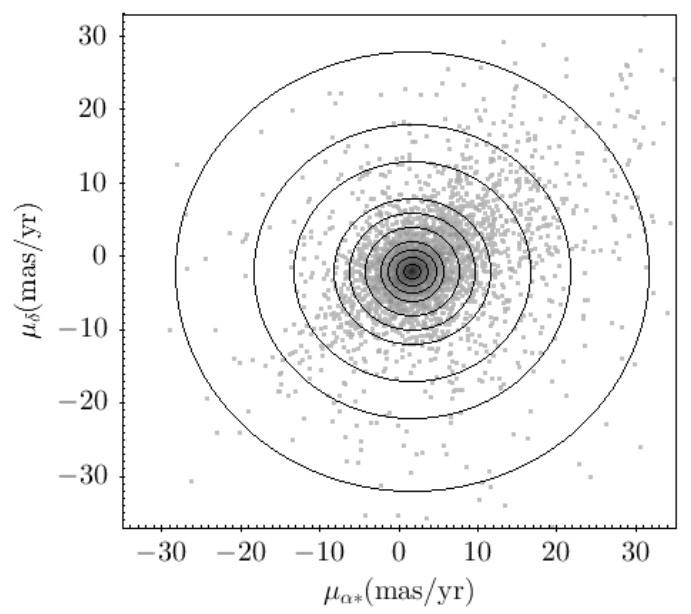

Figure A2. Rings in the VPD of NGC 1261 with increasing proper motions: 0 to $1 \mathrm{mas} / \mathrm{yr}, 1$ to $2 \mathrm{mas} / \mathrm{yr}, 2$ to $3 \mathrm{mas} / \mathrm{yr}, 3$ to $4 \mathrm{mas} / \mathrm{yr}, 4$ to $6 \mathrm{mas} / \mathrm{yr}, 6$ to $8 \mathrm{mas} / \mathrm{yr}, 8$ to $10 \mathrm{mas} / \mathrm{yr}, 10$ to $15 \mathrm{mas} / \mathrm{yr}, 15$ to $20 \mathrm{mas} / \mathrm{yr}$ and 20 to $30 \mathrm{mas} / \mathrm{yr}$.
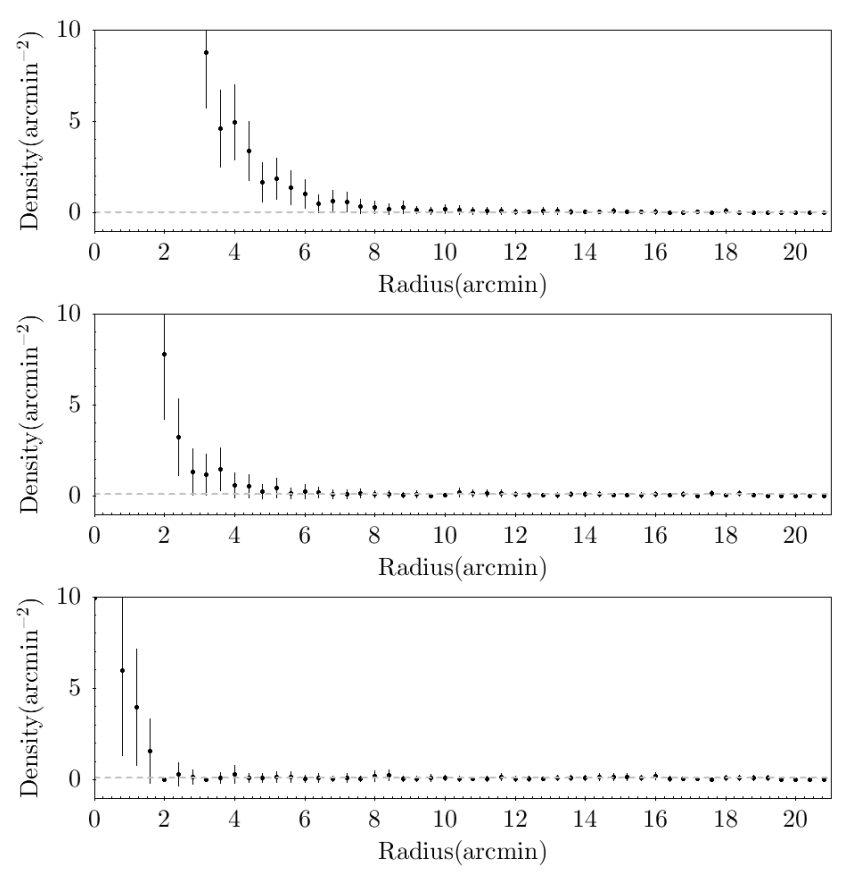

Figure A3. Radial density projected on the sky of all the stars in Gaia DR2 with proper motions in three different rings, with $3 \sigma$ error bars computed assuming Poisson noise in the counts, and horizontal gray dotted line showing the adopted background density in the field of NGC 1261. Upper panel: ring 0 to 1 mas/yr, middle panel: ring 3 to 4 mas/yr, lower panel: ring 20 to 30 mas/yr.

\section{A2 NGC 3201}

In Figure A6 we show residual overdensity and the residual concentration in the VPD around the mean proper motion of NGC 3201. In this case the distribution of the proper motions of the cluster and of the field stars are well separated, a situation like that of NGC 6397 shown in Figure 3.

In the first stage we found 22200 members with mean proper motion $\mu_{\alpha *}=8.3170 \mathrm{mas} / \mathrm{yr}, \mu_{\delta}=-1.9612 \mathrm{mas} / \mathrm{yr}$. We 

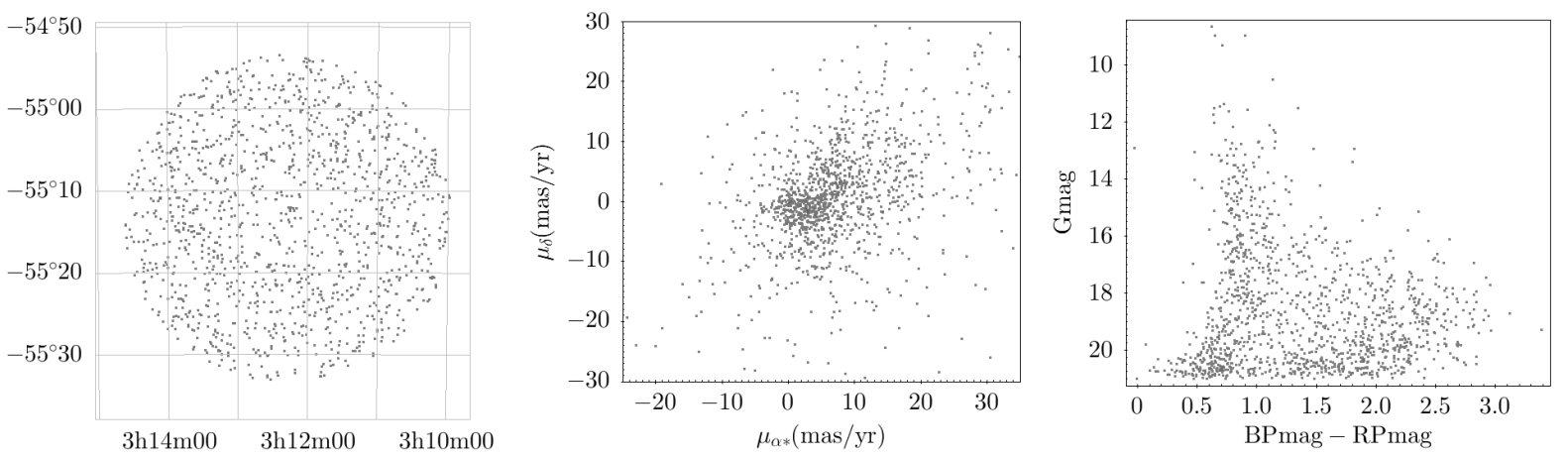

Figure A4. Positions on the field (left), in the VPD (middle) and in the CMD (right) of the field stars after the two stages of extraction of NGC 1261 members.

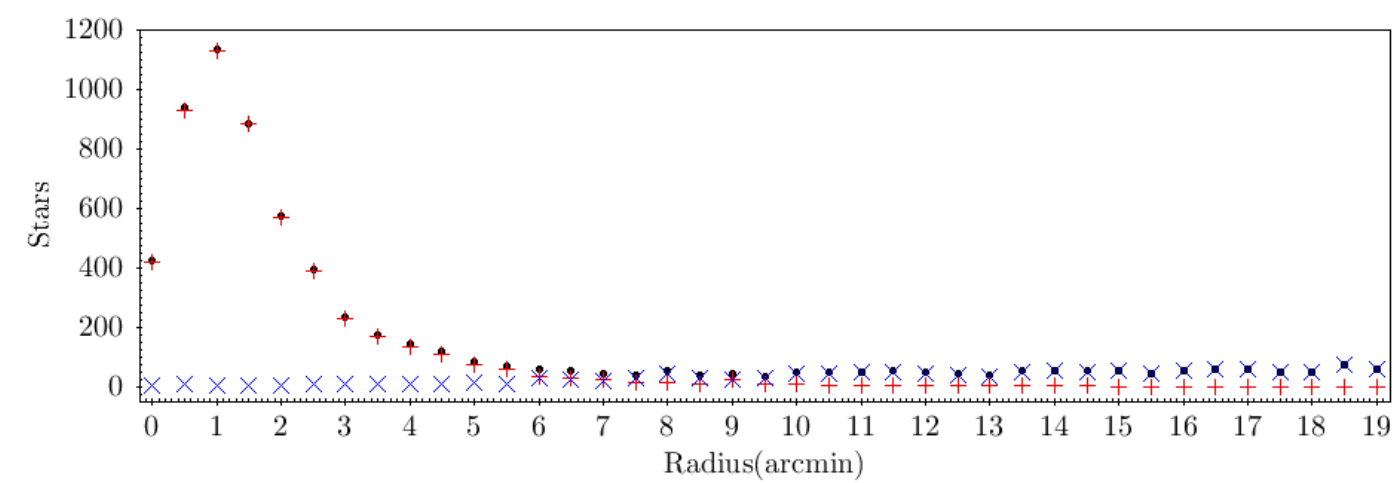

Figure A5. Radial counts of field stars (blue cross), members of the cluster (red plus) and all Gaia DR2 stars with measured proper motions (black dots) in the field of NGC 1261. Notice that most of Gaia DR2 stars in this field are cluster members.
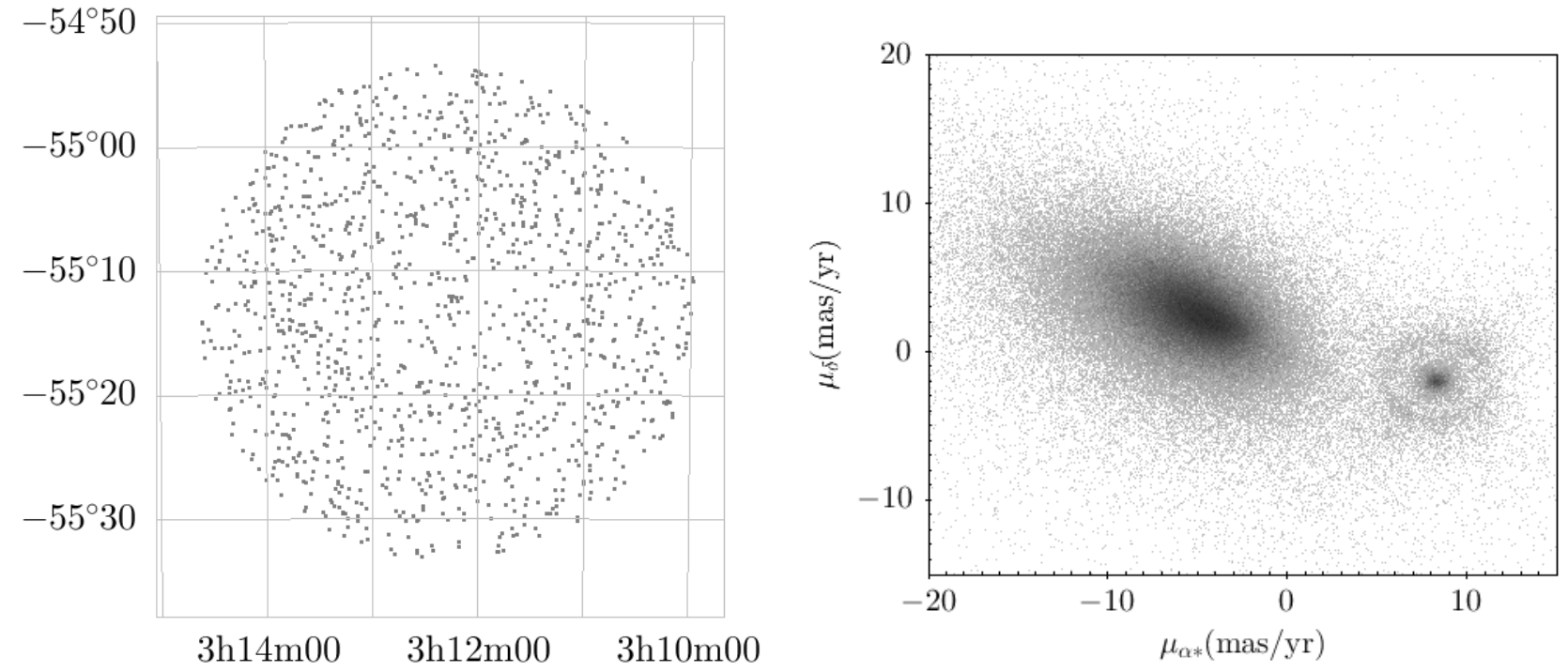

Figure A6. Positions on the field (left) and in the VPD (right) of the stars that were not detected as members of NGC 3201 in the first stage.

selected the stars in Gaia DR2 in rings of proper motions centred in this mean proper motion with increasing radii as shown in Figure A7.

We computed the radial density projected on the sky of all the Gaia DR2 stars within each ring of proper motions, some of which are shown in Figure A8.

From the list of stars that were not classified as mem- bers in the first stage, we select those inside each ring of proper motions that in the field are lying within the radius of the central overdensity corresponding to that ring, and we add them to the list of members of the cluster. As result of this stage 9336 more members were extracted. The distribution of the resulting field stars is shown in Figure A9, where there is no residual overdensity in the field nor resid- 


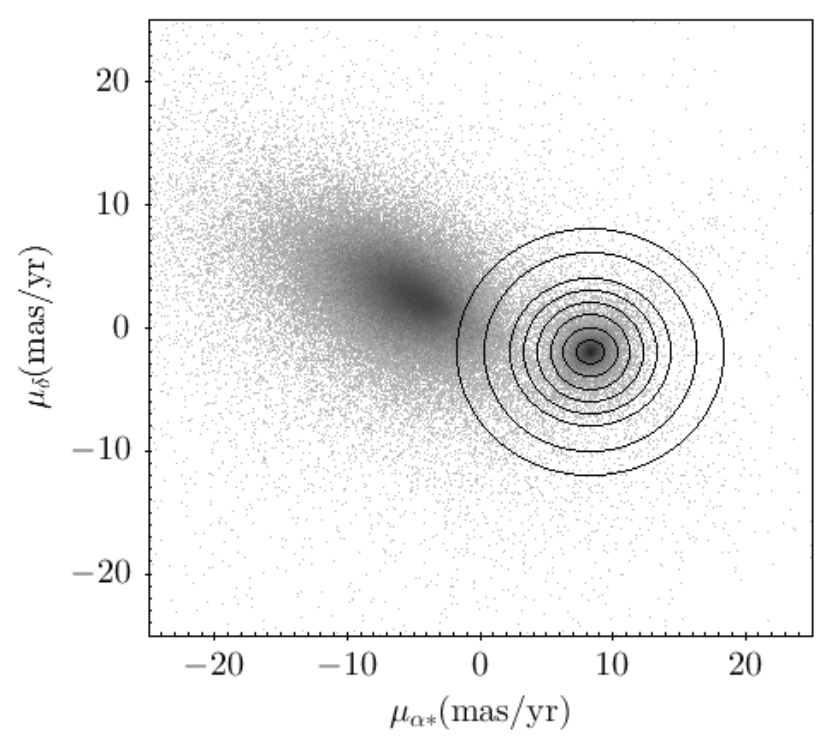

Figure A7. Rings in the VPD with increasing proper motions: 0 to $1 \mathrm{mas} / \mathrm{yr}, 1$ to $2 \mathrm{mas} / \mathrm{yr}, 2$ to $3 \mathrm{mas} / \mathrm{yr}, 3$ to $4 \mathrm{mas} / \mathrm{yr}, 4$ to $5 \mathrm{mas} / \mathrm{yr}, 5$ to $6 \mathrm{mas} / \mathrm{yr}, 6$ to $8 \mathrm{mas} / \mathrm{yr}$ and 8 to $10 \mathrm{mas} / \mathrm{yr}$.

ual concentration in the VPD, and the CMD does not show the typical features of a globular cluster.

As in the previous section (NGC 1261) we performed the radial count of the field stars and we verified that it increases linearly with the radius as expected for a constant density (Figure A10). Note that the radial count of the cluster members is smooth, while the radial count of the field stars is noisier.

This paper has been typeset from a $\mathrm{T}_{\mathrm{E}} \mathrm{X} / \mathrm{LAT}_{\mathrm{E}} \mathrm{X}$ file prepared by the author.
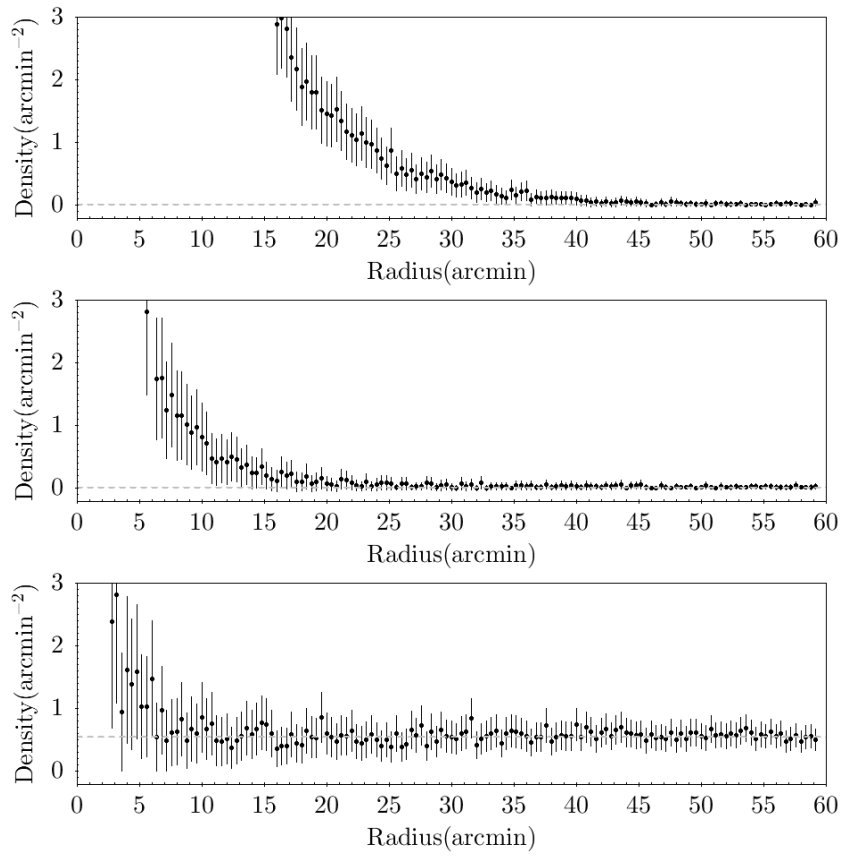

Figure A8. Radial density projected on the sky of all the stars in Gaia DR2 with proper motions in three different rings, with $3 \sigma$ error bars computed assuming Poisson noise in the counts, and horizontal gray dotted line showing the adopted background density in the field of NGC 3201. Upper panel: ring 0 to 1 mas/yr, middle panel: ring 3 to 4 mas/yr, lower panel: ring 8 to 10 mas/yr. 

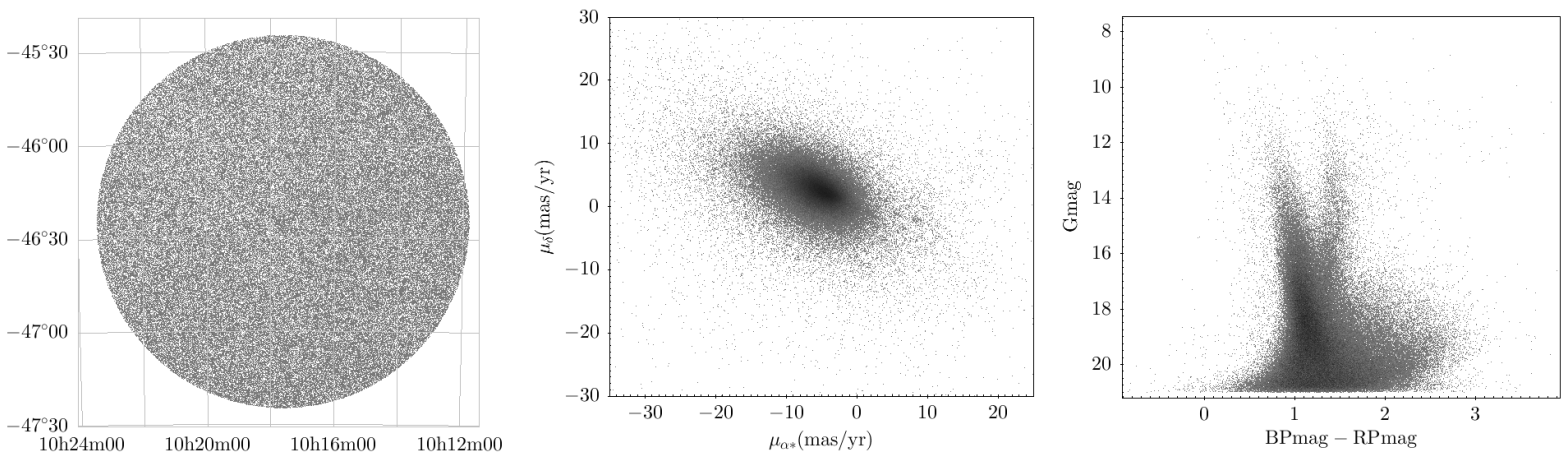

Figure A9. Positions on the field (left), in the VPD (middle) and in the CMD (right) of the field stars after the two stages of extraction of NGC 3201 members.

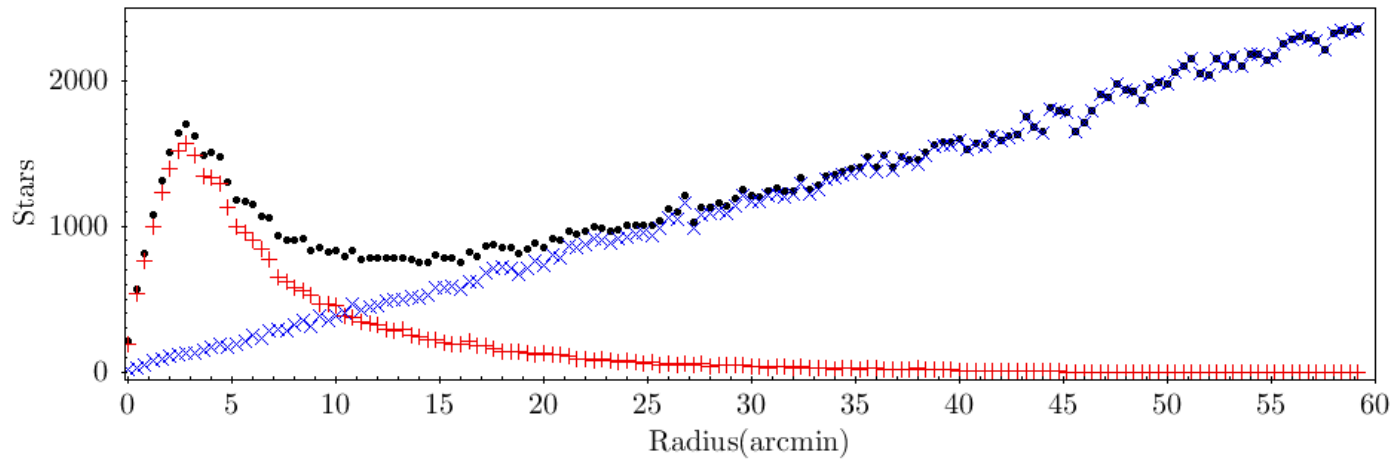

Figure A10. Radial counts of field stars (blue cross), members of the cluster (red plus) and all Gaia DR2 stars with measured proper motions (black dots) in the field of NGC 3201. Notice that most of Gaia DR2 stars in this field are cluster members. 